\title{
Le rewriting et le romanesque dans les reportages de Paris Match (1949-1959)
}

Rewriting and Fiction in Paris Match Reports' (1949-159)

\section{Marie-Ève Thérenty}

\section{OpenEdition}

\section{Journals}

Édition électronique

URL : https://journals.openedition.org/recherchestravaux/3490

DOI : 10.4000/recherchestravaux.3490

ISSN : 1969-6434

Éditeur

UGA Éditions/Université Grenoble Alpes

Édition imprimée

ISBN : 978-2-37747-301-4

ISSN : 0151-1874

Référence électronique

Marie-Ėve Thérenty, « Le rewriting et le romanesque dans les reportages de Paris Match (1949-1959) », Recherches \& Travaux [En ligne], 98 | 2021, mis en ligne le 28 juin 2021, consulté le 09 août 2021. URL: http://journals.openedition.org/recherchestravaux/3490; DOI : https://doi.org/10.4000/ recherchestravaux.3490

Ce document a été généré automatiquement le 9 août 2021.

(c) Recherches \& Travaux 


\title{
Le rewriting et le romanesque dans les reportages de Paris Match (1949-1959)
}

\author{
Rewriting and Fiction in Paris Match Reports' (1949-159)
}

Marie-Ève Thérenty

1 Les spécialistes des relations entre la presse et la littérature qui ont beaucoup travaillé sur les moments et les espaces d'hybridation entre les deux champs ont jusqu'ici laissé de côté les années cinquante, soixante, soixante-dix du vingtième siècle en France, comme si cette époque se caractérisait dans ce pays par une séparation des territoires et des identités avant le retour contemporain des littératures de terrain ${ }^{1}$. En fait l'absence de numérisation des corpus situés entre la couverture opérée par Gallica et celle d'Europresse explique que perdure une méconnaissance de cette presse. Le projet Numapresse ${ }^{2} s^{\prime}$ est justement fondé sur l'hypothèse qu'avaient pu se maintenir dans les hebdomadaires des Trente Glorieuses des formes de littérarisation déjà présentes avant-guerre et reconfigurées par le contexte historique, social et médiatique. Si L'Express, France Observateur et son héritier Le Nouvel Observateur paraissaient dans ce cadre, des pistes fécondes en raison du grand nombre d'écrivains patentés qui y interviennent (François Mauriac, Albert Camus, Roger Nimier, Françoise Sagan, Marguerite Duras...), le cas de Paris Match - magazine avec une réputation fondée sur «le choc des photos", le sensationnalisme, le suivi des célébrités -, pouvait sembler plus douteux. Pourtant ce magazine s'est établi grâce à une pratique assidue du reportage (grand reportage, reportage de guerre, reportage chez les people...) dont le développement dans les années trente en France était lié à l'investissement des écrivains ${ }^{3}$.

2 Le fondateur du magazine est Jean Prouvost, entrepreneur issu d'une grande famille d'industriels du Nord, reconverti durant l'entre-deux-guerres dans la presse. Il relance avec succès en 1930 le quotidien Paris-Soir en y développant le grand reportage et la photographie. En 1936, il rachète un concurrent L'Intransigeant de Léon Bailby et hérite du même coup de Match, le supplément sportif du quotidien. Il le transforme en 
magazine général d'information. Cette première version de Match connaît un énorme succès, atteint un tirage de 1400000 exemplaires en octobre 1939 avant de se saborder avec l'entrée des Allemands à Paris en 1940. À la sortie de la guerre, après des atermoiements dus notamment à la situation personnelle de Jean Prouvost occupé pendant plusieurs années à se défaire d'accusations de collaboration, le magazine Paris Match est relancé le 25 mars 1949 par l'équipe de direction des journaux Prouvost de l'entre-deux-guerres : Pierre Lazareff, Hervé Mille et Philippe Boegner (ancien rédacteur en chef de $V u$ puis de Marie-Claire) mais aussi Gaston Bonheur, Raymond Cartier, Roger Thérond.

3 Le magazine a un tirage de 300000 exemplaires au printemps 1949 réduit à 200000 à la fin de l'année. Le succès arrive enfin le 19 août 1950 avec l'aventure himalayenne d'une poignée d'alpinistes français Maurice Herzog, Louis Lachenal, Lionel Terray et Gaston Rébuffat. Paris Match trouve son public avec le reportage photographique de Marcel Ichac, l'homme des sommets. Notre article se propose de montrer l'omniprésence du reportage dans Paris Match qui se caractérise à la fois comme de juste par un déplacement sur le terrain mais aussi par une couleur, un ton ; en un mot, par un style. Dû à la patte des écrivains-rewriters du magazine, ce style Paris Match se fonde sur un romancement non-fictionnalisant de la matière journalistique.

\section{Un magazine de reportage}

4 Les mémoires des journalistes de la rédaction montrent l'importance essentielle du genre du reportage mobilisé aussi bien pour rendre compte de la vie privée des stars (c'est le début des paparazzi) que pour le reportage de guerre où Paris Match excelle. La forme est tellement prégnante qu'elle nourrit la mythologie du magazine qui se construit sur le double corps du reporter. Le premier corps est sexualisé : les mémoires regorgent de descriptions de reporters photographes aux allures de play-boy et aux postures calquées sur celles de Marlon Brando. Walter Carone, Jean-Pierre Pedrazzini, Willy Rizzo, Jack Garofalo, Pierre Galante - le mari de Olivia de Havilland, significativement surnommé «le reporter marié à son reportage»- et Daniel Filipacchi notamment fréquentent le Paris du cinéma et de la nuit. Plus fondamentalement, le reporter expose aussi son autre corps, celui du martyr au service de l'information. Durant les années cinquante, Paris Match perd deux reporters photographes - Jean-Pierre Pedrazzini, photographe mort en Hongrie le 7 novembre 1956 après un reportage, et Jean Roy, tombé en Égypte le 11 novembre 1956 - et il ne compte pas les blessés comme Jacques de Potier, touché en Indochine, et dont le corps christique de martyr de l'information, les bras en croix, est étalé pleine page ${ }^{4}$.

5 La tradition du reportage littéraire, telle qu'elle a été mythifiée par Albert Londres, Joseph Kessel ou Antoine de Saint-Exupéry, mobilise un imaginaire du grand reportage réalisé sur des terres exotiques. Mais en fait il faut admettre à la lecture du journal que le même personnel pouvait se retrouver en train d'interviewer les stars dans leurs domiciles parisiens comme de crapahuter sur les terrains lointains. Ainsi en novembre 1952, Paris Match envoie dans le bourbier indochinois un duo composé de l'écrivain Philippe de Baleine et du photographe de mode Willy Rizzo. Comme l'avouera Rizzo lui-même plus tard, "on était des zazous, pas des baroudeurs». Entre la chronique de la vie mondaine et le reportage de guerre ne figure aucune rupture: le 
divertissement et la tragédie cohabitent, servis par le même moule générique, celui du reportage.

6 Ce résultat est corroboré par les outils de classification automatisée des rubriques que nous avons fait tourner sur l'ensemble des numéros numérisés par nos soins de la décennie 1949-19595. Le résultat est éclairant: quasiment tous les longs formats de Paris Match sont étiquetés par l'outil de classification TidySupervise comme des reportages. Cette uniformité s'explique facilement par le traitement homogène de l'ensemble des informations: elles sont toutes effectivement récupérées sur le « terrain » par des envoyés spéciaux et habillées ensuite d'une tonalité romanesque par des rewriters. Nous entendons par "romanesque" à la fois les enchaînements de situations (ou de structures d'intrigues) et les affects suscités ou sollicités de manière typique par le genre du roman. Pour le dire avec les mots de Michel Murat, « il s'agit donc d'une catégorie esthétique dérivée, indépendante du genre du roman. Le romanesque consiste à voir la vie comme un roman $^{6}$ ». Et ceci alors même que ne travaillent dans la rédaction de Paris Match que peu de "grands écrivains » (même si on trouve quand même très ponctuellement les signatures de Joseph Kessel, Paul Morand ou François Mauriac). Pour expliquer ce caractère romanesque du reportage, il faut donc revenir sur les conditions de production tout à fait particulières des articles long format chez Match qui font apparaître un personnel essentiel à côté des reporters et des photographes, les écrivains rewriters.

\section{Le Paris Match des rewriters}

7 Paris Match reparaît quatre ans après la Seconde Guerre mondiale, à une époque soucieuse de ne pas réitérer les débordements de l'entre-deux-guerres ${ }^{7}$. Le magazine doit s'employer à une précision extrême et à éviter toute dérive fictionnelle comme le note le groupe $\mu$ en 1970 :

Numériques ou qualitatives, les précisions qui s'étalent dans [...] Paris Match ont une fonction rhétorique banale dans l'écriture de presse. Elles signifient que nous sommes informés de première main par un témoin exceptionnel, le grand Asmodée de la culture planétaire, qui voit tout, entend tout, est partout. Le narrateur forcément anonyme qui nous entretient chaque semaine des héros du jour peut nous décrire la salle de bains d'Henri Langlois en 1936, le mobilier de cuisine de Madame Charden, le bureau de M. Marcel Bleustein-Blanchet, la vue exacte qu'on a sur le paysage quand on se trouve à la direction des nouveaux studios de la « Mosfilm »; l'autre jour, il était en voiture avec la famille Stern (surprenant dans le rétroviseur "un regard de complicité » entre le père et le fils); hier, il déjeunait avec « le père Bruck » au restaurant du Rond-Point, avenue Matignon ; demain, il notera une conversation dans un studio londonien entre Katherine Hepburn et Peter O'Toole ${ }^{8}$.

8 L'écrivain-reporter Jean Diwo raconte dans ses mémoires qu'il avait étudié, avant son entrée au journal, le style Match préconisé par son rédacteur en chef Roger Thérond: "C'est simple, chaque paragraphe doit commencer par une phrase forte, le genre du gong sur un ring, se poursuivre par des informations précises, claires, sans mots inutiles et s'achever sur une image sensible, un peu romantique. Si tu bâtis comme cela ton article, il est bon pour l'imprimerie ${ }^{9}$ ". À l'intérieur de la rédaction, les journalistes se moquent ouvertement de ce style et ne se privent pas de faire courir de savoureux pastiches : 
Les pneus de la DS noire crissèrent sur le gravier, la berline s'immobilisa et Charles de Gaulle en costume gris foncé apparut dans la cour de l'Élysée, tel le commandeur d'un pays très ancien, la Gaule, c'est-à-dire la France ${ }^{10}$.

9 Malgré ce prosaïsme apparent, les anciens reporters de Paris Match évoquent avec une unanimité frappante dans leurs souvenirs une rédaction de "poètes». Guillaume Hanoteau dépeint ainsi le journal comme "l'heureux mariage de quelques gentils voyous et d'une poignée de poètes ${ }^{11}$ ». Michel Clerc utilise exactement la même image, confirmant la mythologie héritière de celle des magazines d'avant-guerre comme Détective ${ }^{12}$ : "L'équipe de Match, dans ces années-là, était un gang étonnant. Moitié héros, moitié voyou, un côté play-boy, un côté poète, nos reporters étaient candidats à tous les coups durs ${ }^{13}$. »

10 Les mémoires dévoilent aussi une rédaction en chef très exigeante sur la qualité textuelle, refusant assez fréquemment des articles, quel que soit le prix qu'ils aient déjà coûté au magazine. La titraille, titres et intertitres, était obligatoirement revue par Gaston Bonheur. Surtout, les longs formats font l'objet d'un rewriting systématique parfois discret, parfois manifeste. En effet, notamment à la fin de la décennie, un grand nombre d'articles de Match sont triplement crédités comme ce très long reportage sur Jules Verne, daté du 11 octobre 1958, où apparaissent, de manière emblématique, les trois énonciateurs : le photographe Willy Rizzo, la reporter Marie Gatard et l'écrivain en charge de l'article Pierre Joffroy, romancier patenté édité au Seuil dans les années 60. Jean Diwo énumère les trois composantes du succès du Paris Match: les "reporters baroudeurs", les "photographes inspirés", deux composantes bien connues, et plus étonnamment : « les écrivains créateurs d'un nouveau journalisme ${ }^{14}$ ", expression évidemment intéressante parce qu'elle sert généralement à qualifier le New Journalism des années 60 aux États-Unis.

11 Tous les témoignages font donc état d'une catégorie spéciale de journalistes au sommet de la hiérarchie du journal : les rewriters appelés aussi reporters-écrivains, chargés de reprendre la prose de tous et de la couler dans le moule Paris Match:

Le Match de la vie qui s'intéressait à la fois à l'actualité internationale, française et parisienne, était écrit de cette façon. Des phrases courtes faisant images. Une multitude de détails parfois accessoires mais toujours exacts et, comme tels, garants de l'exactitude du reste, les phrases prononcées par les acteurs du drame ou de la comédie. Le lecteur devenait un spectateur. On sollicitait autant ses yeux que ses méninges, son imagination que sa réflexion. Un tel journalisme de précision exige le « rewriting ${ }^{15}$ ».

12 Michel Clerc est encore plus précis sur le rôle de ces rewriters chargés non pas seulement d'analyser une situation politique mais «de faire évoluer de vrais personnages dans de vrais décors", d'agencer des détails, bref d'inventer un romanesque sans fiction :

Je suis devenu journaliste parce qu'il n'est pas donné à tout le monde d'être Balzac. [...] La curiosité aidant, j'ai été pendant plusieurs années l'un des hommes à tout faire de Paris Match. Effectivement, j'ai tout « fait » : la cour d'Angleterre, l'Algérie, le Vietnam, le cinéma, les interviews, les grands fleuves, les faits divers, le Quai d'Orsay, le Quai des brumes, la maison Blanche, les maisons closes, le show Business et le business, les starlettes et les chefs d'état. Il existait, pour désigner ma fonction, un titre ronflant: "grand reporter écrivain ». Cette précision signifiait qu'à la différence des reporters tout court j'étais capable non seulement de chasser l'information, mais d'écrire mes propres articles, et même ceux des autres ${ }^{16}$ 
Philippe Joffroy, Georges Reyer, Michel Clerc, Jean Maquet, Jean Farran, Jean Diwo, Philippe de Baleine étaient les rewriters efficaces de Paris Match dans ces années-là... Or sans être des écrivains légitimés par l'histoire littéraire, ces rewriters ont été des auteurs prolifiques. Il faut cependant remarquer, dans le cas de Paris Match, quelques points de rupture, avec la tradition d'une presse magazine fréquentée, déjà dans l'entre-deux-guerres, par les écrivains. Les rewriters de Match n'ont généralement pas le temps de produire une œuvre lorsqu'ils sont salariés par le magazine. Certains ont eu une carrière antérieure dans la littérature comme Georges Reyer, lauréat en 1937 du Prix de la Renaissance pour Le Magasin de travestis (Gallimard) ou Jean Maquet, transfuge étonnant, professeur de philosophie fondateur de la revue Le Troisième convoi à laquelle ont contribué Georges Bataille, Francis Picabia, Georges Lambrichs... Les autres se sont révélés, après leur passage à Paris Match, des écrivains de best-sellers comme Michel Clerc, auteur de Backchich (Flammarion, 1976) ou Jean Diwo, à la tête d'une abondante série, Les dames du faubourg chez Gallimard, qui s'écoule, à partir de 1985, à plus d'un million d'exemplaires. Le journaliste Philippe de Baleine, inspirateur du personnage de Jean-Loup de la Batellerie dans Les Bijoux de la Castafiore, est un écrivain invisible, auteur notamment, sous le pseudonyme de Margaret Ring, d'une vingtaine de romans policiers...

\section{Les romanesques de Paris Match}

14 Même si c'est le reportage chez les stars et les princesses qui est le plus fréquemment stigmatisé par les détracteurs de Paris Match, l'ensemble du rédactionnel peut être identifié comme du reportage " romancé ». Tous les genres de reportages - reportage people, grand reportage et reportage littéraire - fonctionnent selon les mêmes ressorts, quels que soient les sujets : l'émotion de la reconnaissance du déjà lu, l'impression d'un accroissement des capacités cognitives, et le plaisir d'une forme d'esthétisation du réel. L'intertexte de référence, romance ou novel selon la classification britannique qui distingue ainsi la littérature d'imaginaire et la littérature réaliste, peut différer selon les cas et le système d'interprétation peut se complexifier, comme nous le verrons, mais toujours est-il que le romanesque est, à chaque fois, convoqué pour les mêmes raisons : le divertissement, la mobilisation empathique, la vraisemblabilisation et une forme de jeu, de connivence sur la reconnaissance de la référence.

\subsection{Le romanesque romance}

Les vilipendeurs de Paris Match dénigrent surtout le romanesque des stars, résolument transmédiatique et emprunté à la fois à la littérature et au cinéma :

C'est un maire de village, ceint de l'écharpe tricolore, qui va déclarer unis par les liens du mariage Rita Hayworth, étoile, et Ali Khan, prince des mille et une nuits. Jusque là, toutes les conventions qui font les belles histoires d'amour avaient été respectées : la beauté, la fortune, les Indes mystérieuses, les sortilèges d'Hollywood, la toile de fond de la Méditerranée. Il n'y manquait même pas le hasard (une fée distraite) qui fit s'asseoir un soir le prince Ali à côté de Rita à une même table où ils allaient boire, sans le savoir, le philtre des légendes. Et tout cela, qui n'aurait pu être que l'argument d'un film en technicolor courant à l'apothéose, s'achève à la Pagnol dans le style gentil des noces de village ${ }^{17}$. 
16 Après le roman de «l'hérö̈ne la plus romanesque de notre temps ", la superlative Rita, le lecteur peut se plonger dans le roman d'Ingrid car toutes ces actrices sont uniquement prénommées comme si leur starisation les condamnait à devenir intimes de l'ensemble de la nation. Le journal, à la suite d'une lutte épique de paparazzi pour l'obtention des photographies du bébé Rossellini, l'enfant d'Ingrid Bergman et de Roberto Rossellini, se sentant tenu de se justifier, explique que lorsqu'une histoire atteint le niveau d'une universelle romance, elle devient évidemment prioritaire sur le respect de la vie privée :

Il n'y a rien de malsain dans cette demande et ce serait faillir au souci de rapporter la vie du monde que de se condamner à ignorer les héros du cœur. Quand une aventure, quelle qu'elle soit, atteint au retentissement mondial, on peut affirmer qu'elle échappe au fait divers pour entrer dans la légende. Chaque époque a ses Tristan et Yseult. C'est la perpétuelle incarnation du mythe éternel de l'aventure amoureuse. C'est si vrai qu'au-delà des personnes, le duo Bergman-Rossellini sousentend un duo épique Amérique-Latinité. La morale, les morales jugent et prennent parti. Un grand hebdomadaire d'information ne peut mésestimer le cœur. Il a ses raisons, auxquelles répond un article comme celui-ci qui s'efforce d'aborder avec l'objectivité du psychologue (et l'objectif du photographe) un problème par définition privé mais dont le public a choisi de faire une romance internationale et publique. Témoigner n'est pas juger ${ }^{18}$.

17 Les princesses constituent l'autre personnel de ce romanesque à l'eau de rose. Dans ce registre, la Cour d'Angleterre constitue l'espace de prédilection où s'ébat Paris Match qui a le recours à la fois de la reine Elisabeth, présentée la plupart du temps comme à l'origine d'un romanesque ordinaire malgré sa couronne - «Le Roman de toutes les femmes écrit avec l'encre de l'histoire ${ }^{19}{ }$ - et de la princesse Margaret au cœur, elle, d'un roman extraordinaire puisqu'elle est contrainte par l'étiquette à renoncer à l'aviateur Peter Townsend. Paris Match ne boude pas son plaisir devant «le climat de suspense dans ce film rose aux rebondissements mystérieux ${ }^{20} »$. Cette double figure de la cour d'Angleterre renvoie aux deux versants du romanesque et de Match - d'un côté l'imagination, le rêve et l'évasion et de l'autre le vraisemblable, le quotidien, le typique - et montre bien leur proximité, voire souvent leur coïncidence.

Mais le romance se retrouve aussi dans le reportage criminel fortement contaminé par le roman policier. Rabaudy ainsi raconte dans son ouvrage sur Paris Match que la rédaction leur donnait volontiers Georges Simenon comme modèle. Visiblement tous obtempèrent avec docilité, ne manquant pas d'indiquer par un repère la place de l'intertexte :

Le juge souriant [...] a perdu le sourire. Les mystères d'Arras étaient trop lourds pour lui. Dimanche, il est passé par la fenêtre. Mais Arras refuse le scénario à la Simenon que le destin lui impose depuis un $\mathrm{an}^{21}$.

19 Comme dans un roman de Simenon, le juge d'instruction Fougères écrivit avec soulagement « à classer » sur le dossier ${ }^{22}$.

Sous les éclats du soleil, Port-Joinville avec ses maisons passées à la chaux et soulignées de bleu devait être une charmante petite ville. Dans les buées fades de l'aube, ce quai qu'éclairait encore l'arc-en-ciel des réverbères, ressemblait à un décor de Simenon ${ }^{23}$.

La place importante réservée par le magazine à Simenon ne vient donc pas seulement du grand nombre de reportages auprès de l'écrivain, bon client de l'hebdomadaire, mais aussi du grand nombre d'articles où il sert de balise du romanesque. 
21 En fait chaque genre romanesque a son pendant journalistique et tout événement réel peut être reformulé à travers la convention d'intertextes fictionnels; certains reportages sur l'aéropostale, les navigateurs en solitaire, la Légion brassent explicitement les codes du roman d'aventures, d'autres articles convoquent le roman d'espionnage au moment même où Paris Match publie en feuilletons Le Troisième homme de Graham Greene. Tous ces exemples pourraient laisser croire que le romanesque habille uniquement les nouvelles à scandale ou à sensation, pour séduire « la concierge de Jean Prouvost », personnage décisif dans les choix rédactionnels si l'on en croit les mémoires des journalistes. En fait, le romanesque est partout dans le magazine et il fonctionne aussi comme une clé des articles les plus informés, par exemple ceux de Raymond Cartier, l'éminence grise du magazine.

\subsection{Le romanesque novel}

Dans les articles à la tonalité grave, comme certains reportages de guerre ou dans les reportages politiques de Cartier, le plus souvent l'article mobilise les modes d'écriture du roman réaliste fondés sur l'intervention d'un personnage typique inséré dans un décor adéquat et un récit fondé sur l'enchaînement d'événements organisés selon la topique de la vraisemblance. Comme dans le romanesque romance, le journaliste signale la mobilisation par l'emploi d'une isotopie explicite autour de roman/romancier/ romanesque comme ici dans cette description du légionnaire qui comprend beaucoup d'indices du romanesque avant de bifurquer vers le type :

C'est la légende de la Légion - pleine d'héritages fabuleux, de coups de foudre, de noubas à tout casser et de barouds fantastiques - qui exécute sa danse de bayadère devant les « anciens » noyés de souvenirs et les « bleus » avides d'aventures.

Cette danse, personne ne sait la conduire comme le caporal Pavel qui a tiré toutes les bordées, a été de tous les coups durs et connaît plus d'histoires que Schéhérazade. Grand, très beau, sanglé dans un drap d'officier, le képi blanc un peu sur l'oreille et ganté de cuir fauve, le caporal Pavel, qui a un œil écorché, est le légionnaire dont rêvent toutes les midinettes et qui fascine les «bleus » avides d'aventure ${ }^{24}$.

Un autre procédé pour installer l'horizon d'attente consiste à inviter explicitement un écrivain dit réaliste $d u \mathrm{XIX}^{\mathrm{e}}$ siècle. Le plus souvent sont convoqués, comme le prouve l'extraction par des moyens automatisés des entités nommées, Stendhal et Balzac:

Stendhal dont on dit que le Verrières de Julien Sorel est Salins à quelques kilomètres de Port-Lesney, fief d'Edgar, aurait aimé la manière dont l'avocat parisien a fait la conquête du département. Il en aurait aimé les personnages étonnants : le doux évêque affable et habile Mgr Flusin, M. Poux, ce négociant en vins d'Arbois embarrassé de ses 12000 voix, M. Celas le bras droit d'Edgar qui a peur de se faire attraper par sa jeune et jolie femme ${ }^{25}$...

Les habitués de la bourse, tous ces personnages étranges sortis des romans de Balzac ou des dessins de Daumier, tous ces petits vieillards négligés et mystérieux qui dans l'ombre des escaliers troquent leurs secrets s'étaient arrêtés de parler ${ }^{26}$.

En fait, la convocation de l'écrivain ou des modes de narrations romanesques produit un effet de vraisemblabilisation. Ces intertextes apportent au texte journalistique une sorte de cohérence extérieure. Ce recours à la vraisemblance romanesque permet d'appuyer le texte sur une série d'attentes et la fiction est mobilisée comme validation du réel. reconnaissance de la référence, un effet esthétique. Sur le principe, le romanesque novel 
ne se différencie donc pas fondamentalement du romanesque romance (Balzac et Simenon ou Pagnol, même combat), mais la distribution des effets recherchés diffère sans doute. Alors que pour le texte d'information générale, l'effet de validation prime sur l'effet empathique; pour le texte people c'est probablement le contraire, mais dans les deux cas, une émotion esthétique, qui tient aussi de la connivence culturelle et sociale, se déclenche à la reconnaissance de l'intertexte.

La division entre romanesque romance et romanesque novel paraît donc presque artificielle tant la même rhétorique traverse tout le journal. Depuis les mystères d'Arras, un fait divers, jusqu'au " plus grand mystère commercial du siècle ", article sur Coca-Cola, en passant par " un roman sous chaque képi blanc ${ }^{27}$ ", reportage de guerre en Algérie, c'est le même "romanesque général» (pour reprendre les termes de Marc Angenot ${ }^{28}$ ) ou "mystère général " (pour citer Filippos Katsanos, qui voit dans cette forme de sensationnalisme cognitif la recette médiatique sur laquelle s'élaborent à la fois la culture dite populaire mais aussi le système informationnel ${ }^{29}$ ), catégories appliquées pour décrire le système médiatique $d u$ xix ${ }^{e}$ siècle mais qui perdurent visiblement dans le Match des Trente Glorieuses.

\subsection{Le romanesque des lettres}

Évidemment ce romanesque s'exprime de manière particulièrement exacerbée et spéculaire dans les articles qui traitent de la littérature. Organisés autour de l'homme plutôt que de l'œuvre, ces très nombreux reportages aux domiciles d'écrivains vivants remplacent dans le magazine la critique littéraire. Ils convoquent « le romanesque des lettres ", c'est-à-dire une propension à lire tout ce qui forme et transmet la littérature comme un roman ${ }^{30}$. Les mêmes procédés sont utilisés que pour les articles précédents mais, en raison du terrain même de leur exercice, le romanesque des lettres de Paris Match produit de somptueux effets de diffraction entre fiction et réel. Se met en place à ce moment-là une forme d'écriture de la littérature qui triomphe encore aujourd'hui: le storytelling d'écrivain. Le romanesque dans ces articles est souvent interprété comme la propre production fictionnelle de l'écrivain si bien que, par exemple, la biographie croise la vie des personnages des héros au prix de métalepses où l'écrivain apparaît comme fréquentant ses propres personnages. Ici, dans un article au titre significatif « $\mathrm{Au}$ coin d'une rue de Liège, la gloire et Maigret attendaient Simenon » :

Pendant ces deux heures frénétiques, il entre littéralement en transes. On prétend qu'il participe à la vie de ses personnages, au point d'accomplir leurs gestes. Lorsqu'ils boivent, il boit. Lorsqu'ils sont malades, il se bourre de cachets. Il n'est pas rare qu'il brise sa machine à écrire en composant des scènes de violence ${ }^{31}$.

Un article sur André Maurois constitue un cas d'école avec des effets spéculaires de renvoi entre le romanesque et la vie que l'on peut énumérer en admirant la manière dont ils se superposent et dialoguent entre eux. D'abord Maurois se comporte comme un héros de roman. Il rencontre sa première femme selon le principe du roman d'amour ou du romanesque romance:

Un beau jour, elle s'enfuit et vint le rejoindre à Paris où il « descendait » deux jours par semaine pour traiter des affaires de drap. Maurois fit alors la chose la plus charmante, la plus touchante et la plus romanesque. Jugeant que cette jeune fille méritait un délai et qu'un mariage trop rapide la désorienterait, elle si jeune, il l'envoya en Angleterre, à ses frais, passer deux ans à Oxford où il alla la voir chaque dimanche en prenant le paquebot de nuit ${ }^{32}$. 
la rencontre avec sa deuxième femme se construit selon le principe du romanesque novel comme le suggère la convocation d'un nouvel intertexte qui montre Proust dans la peau d'un rewriter Paris Match :

Lors de leur première rencontre, Simone de Caillavet raconta à Maurois que Proust la peignit sous les traits de Mademoiselle de Saint-Loup ${ }^{33}$.

L'ensemble finit par produire un bel effet «romanesque des lettres » avec une splendide métalepse.

Je regardais, dit Maurois, avec émotion, une femme vivante qui était un peu Mademoiselle de Saint-Loup ${ }^{34}$.

(1) Maurois s'assimile à Stendhal, référence qui vient se mêler abruptement selon le principe de la transfictionnalité ou du crossover, avec la comparaison de Philippe de Baleine, le portraitiste qui lui le voit en "Sherlock Holmes des lettres ». Là encore selon le principe de la combinaison ou du collage caractéristique de la culture post-moderne, le journaliste brasse des niveaux très hétéroclites de références.

L'illustration enfin assimile l'ensemble du reportage, comme l'a déjà remarqué plus généralement Philippe Marion $^{35}$, à un roman-photo. Dans ces articles, le romanesque est saisi comme une catégorie problématique et fascinante, sans qu'il y ait la moindre ironie. La fin de l'article se termine par l'énoncé d'un nouveau niveau : le romanesque médiatique qui finit par affecter tout écrivain ou toute personne publique :

Il est certain que chaque grand écrivain à succès traîne après lui un "Personnage ", caricature de lui-même, construction extravagante de la calomnie ${ }^{36}$.

Dans un article sur Julien Green, une effarante confession, en écho à ce romanesque médiatique, montre l'écrivain soudain saisi par la peur d'avoir quitté la vie pour l'imaginaire :

Un jour, en 1916, Julien Green était assis dans un cinéma de la rue Cernuschi. Sur l'écran on projetait des actualités ayant trait à la guerre. Tout à coup, Green eut la sensation qu'il ne vivait pas mais qu'il rêvait, que tout ce qui l'entourait n'était pas réel mais imaginaire. Pour chasser le malaise qui l'étreignait, Julien dut dans l'obscurité prendre dans sa poche un papier et écrire "non je ne rêve pas ${ }^{37}$ ".

Ces formes assez fascinantes n'apparaissent pas dans l'histoire littéraire du journalisme narratif français, telle qu'elle a été élaborée jusqu'à présent, qui saute assez aisément, comme en témoignent le travail de Géraldine Muhlmann ${ }^{38}$ ou la thèse de Marie Vanoost ${ }^{39}$, du reportage littéraire des années 30 au reportage du Libé des années 70, comme s'il n'y avait pas un maillon intermédiaire assumant la tradition du reportage littéraire français. L'examen de Paris Match avec son personnel affecté invite à fortement revenir sur cette idée. L'invisibilité de ces corpus s'explique par l'absence de mise en recueil, absence qui se justifie par l'écriture très collective qui est le soubassement du reportage. Il reste aussi à étudier dans la décennie suivante comment cette esthétique se modifie au contact des écritures du nouveau journalisme américain. Dès ces années 50, Truman Capote par exemple est bien identifié par Paris Match et présent à Paris, il donne des textes à Marie-Claire qui fait partie du groupe Prouvost.

La médiapoétique de presse est une étude poétique des textes journalistiques qui prend en compte leur médium de production, les canaux qu'ils empruntent, leurs visées pragmatiques, leurs enjeux sociétaux, leurs reprises, leurs dérivations, leurs circulations. Ce type d'analyse n'envisage pas d'étudier le fonctionnement interne des articles et des rubriques en microanalyse sans appréhender l'équilibre des rubriques à 
l'intérieur d'un journal, et même le positionnement d'un médium dans des séries culturelles et médiatiques en diachronie ou en synchronie. En termes de médiapoétique, en fait ces nombreux articles composés de romanesque rewrité finissent par venir remplacer le roman-feuilleton, qui s'éteint après quelques années, de Paris Match. Toute une série d'objets hybrides, identifiés par l'explorateur de Numapresse comme des romans, mais qui sont en fait principalement du reportage rewrité, constituent aussi du substitut romanesque. Le relais du feuilleton par un romanesque formé de journaux intimes romancés, de pièces de théâtre narrativisées, de films novellisés, de romans résumés et surtout de reportages rewrités fait de Paris Match une compilation extraordinaire des nouvelles formes du romanesque. La case feuilleton perdure dans le magazine à travers tous ces ersatz.

\section{NOTES}

1. Sur ce dernier point, voir par exemple Laurent Demanze, Un nouvel âge de l'enquête, portraits de l'écrivain contemporain en enquêteur, Paris, José Corti, 2019.

2. Suivant la méthodologie que l'histoire littéraire renouvelée de la presse préconise depuis quelques années, cet article repose à la fois sur l'immersion dans le magazine et sur la lecture la plus exhaustive possible des articles, conjuguées avec l'utilisation des outils d'exploration des corpus de presse numérisés mis en place par le projet Numapresse.

3. Myriam Boucharenc, L'Écrivain reporter au cœur des années trente, Villeneuve-d'Ascq, Presses universitaires du Septentrion, 2004 et Mélodie Simard-Houde, Le Reporter et ses fictions. Poétique historique d'un imaginaire, Limoges, Presses universitaires de Limoges, coll. « Médiatextes », 2017.

4. Voir Jean Roy, «Croix de guerre à notre envoyé spécial sur le front de Na-Sam », Paris Match, $\mathrm{n}^{\circ} 200,10$ janvier 1953, p. 13.

5. Pour plus d'informations sur ces outils mis en place par Pierre-Carl Langlais, voir <http:// www.numapresse.org/>.

6. Michel Murat, Le Romanesque des lettres, Paris, José Corti, 2017, p. 12.

7. Sur ces débordements nous renvoyons par exemple à Roy Pinker, Faire sensation. De l'enlèvement du bébé Lindbergh au barnum médiatique, Marseille, Agone, 2017.

8. Jacques Dubois, Francis Edeline, Jean-Marie Klinkenberg, Philippe Minguet, François Pire, Hadelin Trinon, «Les biographies de Paris Match », Communications, n 16, 1970, p. 123.

9. Jean Diwo, Le Jeune homme en culotte de golf, Paris, Flammarion, 2008, p. 133.

10. Nicolas de Rabaudy, Nos fabuleuses années Paris Match, Paris, Scali, 2007, p. 152.

11. Guillaume Hanoteau, La Fabuleuse histoire de Paris Match, Paris, Plon, 1976, p. 41.

12. Sur Détective, nous nous permettons de renvoyer à Amélie Chabrier et Marie-Ève Thérenty, Détective, fabrique de crimes, Nantes, éditions Joseph K, 2017.

13. Michel Clerc, Tapis rouge, Paris, Julliard, 1998, p. 20.

14. Jean Diwo, Le Jeune homme en culotte de golf, ouvr. cité, p. 184.

15. Hervé Mille, Cinquante ans de presse parisienne ou la nuit du Strand, Paris, La Table ronde, 1992, p. 188.

16. Michel Clerc, Tapis rouge, ouvr. cité, p. 16.

17. «Le roman d'amour de Rita et d'Ali », Paris Match, n 9, 2 mai 1949, p. 27. 
18. «Ingrid Rossellini est-elle plus heureuse qu'Ingrid Bergman », Paris Match, $n^{\circ}$ 65, 17 juin 1950, p. 11.

19. «Elisabeth, femme inconnue », Paris Match, $\mathrm{n}^{\circ}$ 55, 5 avril 1950, p. 26.

20. "Ses vingt-cinq ans apportent à Margaret la liberté du cœur ", Paris Match, n 335,27 août 1955 , p. 59.

21. "Arras, "Capitale du mystère" ", Paris Match, $\mathrm{n}^{\circ}$ 77, 9 septembre 1950, p. 4.

22. Jean Diwo, « Trois Français portent un nouveau coup au B.K », Paris Match, $n^{\circ}$ 315, 9 avril 1955, p. 74.

23. Guillaume Hanoteau, "L'infirmière de l'île d'Yeu se souvient ", Paris Match, n 354, 31 mars 1956, p. 81.

24. Georges Reyer et Izis, "Un roman sous chaque képi blanc », Paris Match, n 223, 27 juin 1953, p. 34.

25. "Jean Farran vous raconte les drames et les comédies d'une campagne électorale comme la vivent 90 départements. Sur les pas de l'Edgar », Paris Match, n 351, 31 décembre 1955, p. 32.

26. "Jean Farran vous fait vivre les heures dramatiques du krach de Paris ", Paris Match, $\mathrm{n}^{\circ} 322$, 28 mai 1955 , p. 17.

27. Georges Reyer et Izis, " Un roman sous chaque képi blanc », Paris Match, n 223, 27 juin 1953.

28. Marc Angenot, 1889, un état du discours social, Montréal, Le Préambule, 1989.

29. Filippos Katsanos, La Littérature des mystères. Poétique historique d'un succès médiatique du XIX ${ }^{e}$ siècle en France, en Grèce et en Grande-Bretagne, Limoges, PULIM, 2020.

30. Michel Murat, Le Romanesque des lettres, ouvr. cité, p. 7.

31. Philippe de Baleine, «Au coin d'une rue de Liège, la gloire et Maigret attendaient Simenon », Paris Match, $\mathrm{n}^{\circ}$ 165, 17 mai 1952, p. 36.

32. Philippe de Baleine, "André Maurois, l'académicien romanesque ", Paris Match, $\mathrm{n}^{\circ} 282$, 21 août 1954, p. 26.

33. Ibid.

34. Ibid.

35. Philippe Marion, "Propositions pour une médiatique narrative appliquée. Lecture d'un reportage photographique de Paris Match ", Jan Baetens et Ana Gonzalez Salvador (dir.), Le Romanphoto, actes du colloque de Calaceite (fondation Noesis), p. 156-180.

36. " André Maurois, l'académicien romanesque », op. cit.

37. Guillaume Hanoteau, «Julien Green porte à la scène le diable qu'il a connu enfant dans le placard de sa mère ", Paris Match, n 258, 6 mars 1954, p. 60.

38. Géraldine Muhlmann, Une histoire politique du journalisme, $\mathrm{XIX}^{e}-\mathrm{XX}{ }^{e}$ siècles, Paris, P.U.F., 2004.

39. Marie Vanoost, Le Journalisme narratif aux États-Unis et en Europe francophone: modélisation et enjeux éthiques, thèse soutenue à l'université Louvain, 2014.

\section{RÉSUMÉS}

Cet article se propose de montrer l'omniprésence du reportage dans le Paris Match de la décennie 1949-1959. Le reportage, qui se définit d'abord par le déplacement d'un reporter sur le terrain, se caractérise aussi par une uniformisation textuelle. Elle est due au romancement non fictionnalisant de la matière journalistique opéré par les écrivains-rewriters du magazine. L'article montre que cette opération à l'ambition autant communicationnelle qu'esthétique 
s'effectue quel que soit le genre de reportage : reportage people, reportage de guerre, reportage littéraire.

This article proposes to show the omnipresence of the reporting in the Paris Match of the decade 1949-1959. The report, which is first defined by sending a journalist into the field, is also characterized by textual standardization. It is due to the non-fictionalizing novelization of the journalistic material operated by the magazine's rewriters. The article shows that this operation, which has both communicational and aesthetic ambitions, is carried out regardless of the type of report: celebrity report, war reporting, literary report.

\section{INDEX}

Mots-clés : magazines, reportage, fiction

Keywords : magazines, reports, fiction

\section{AUTEUR}

\section{MARIE-ÈVE THÉRENTY}

Marie-Ève Thérenty est professeure des universités et directrice du centre de recherche RIRRA 21 à l'université Paul Valéry-Montpellier 3. Spécialiste des rapports entre presse et littérature, de poétique des supports et d'imaginaire des sociétés médiatiques, elle a publié plusieurs ouvrages dont Mosaïques. Être écrivain entre presse et roman (1829-1836) (Champion, 2003), La Littérature au quotidien. Poétiques journalistiques au XIX ${ }^{e}$ siècle (Seuil, 2007) et Femmes de presse, femmes de lettres. De Delphine de Girardin à Florence Aubenas (CNRS Éditions, 2019). 